\title{
Trapping Neutral Molecules in a Traveling Potential Well
}

\author{
Hendrick L. Bethlem, Giel Berden, André J. A. van Roij, Floris M. H. Crompvoets, and Gerard Meijer \\ FOM-Institute for Plasmaphysics Rijnhuizen, P.O. Box 1207, NL-3430 BE Nieuwegein, The Netherlands, \\ and Department of Molecular and Laser Physics, University of Nijmegen, Toernooiveld 1, NL-6525 ED Nijmegen, The Netherlands
}

(Received 11 February 2000)

A series of pulsed electric fields can be arranged such that it creates a traveling potential well in which neutral dipolar molecules can be confined. This provides a method to transport, to decelerate, and to cool a sample of neutral molecules while maintaining the initial phase-space density. This method is described using the concept of phase stability. The oscillating motion of molecules in the traveling potential well, reaching a minimum velocity spread corresponding to a translational temperature of $4 \mathrm{mK}$, is experimentally observed.

PACS numbers: 33.80.Ps, 33.55.Be

Cooling and trapping of atoms has led to numerous exciting results during the last decades [1]. Triggered by this, there currently is a large interest in schemes to produce ultracold molecules [2-5]. Laser cooling has been successfully applied to obtain high densities of cold atoms, starting from a thermal beam or background gas; spontaneous emission acts as a friction force, providing a method to increase the initially rather low phase-space densities. However, laser cooling cannot easily be applied to molecules since off-resonant decay to different vibrational levels in the electronic ground state hampers efficient momentum transfer.

Adiabatic cooling in an expansion can be used to obtain high densities of cold molecules. In pulsed molecular beams, densities on the order of $10^{12}$ molecules $/ \mathrm{cm}^{3}$ in a single quantum state at translational temperatures of $1 \mathrm{~K}$ are readily achieved. Typical velocities in a molecular beam are in the $250-2000 \mathrm{~m} / \mathrm{s}$ range, however, preventing trapping of these dense low-temperature samples in the laboratory frame. Various methods are currently being explored to translate the high phase-space densities from the moving frame of the molecular beam to the laboratory frame [6-9]. These molecules can then be further cooled using evaporative cooling or using laser cooling on rovibrational transitions.

Static electric and magnetic fields have been used to deflect and focus polar and paramagnetic molecules since the 1920s. Already in the 1950s it was realized that timevarying electric fields can be used to change the longitudinal velocity of polar molecules $[10,11]$. Molecules possessing an electric dipole moment will gain Stark energy upon entering an electric field when in the appropriate quantum state. This gain in Stark energy ("potential" energy) is compensated by a loss in kinetic energy. If the electric field is greatly reduced before the molecule has left the electric field the molecule will not fully regain the lost kinetic energy. This process may be repeated by letting the molecules pass through multiple pulsed electric fields. Molecules can thus be slowed down and eventually brought to a standstill. Recently, we successfully demonstrated deceleration of dipolar molecules using an array of pulsed electric fields [6]. Time-varying electric [7] and magnetic [12] fields have also been used to manipulate atoms released from a magneto-optical trap, as well as to change the velocity of a neutron beam [13].

The possibility to manipulate both the transversal and longitudinal velocities of neutral dipolar molecules allows one to perform all the operations on these molecules that are successfully used throughout on charged particles. In this Letter we demonstrate that neutral dipolar molecules can be transported, decelerated, and cooled employing the principle of phase stability. This principle, discovered independently by Veksler [14] and McMillan [15], forms the basis for synchrotronlike charged particle accelerators and can be viewed as trapping of the particles in a traveling potential well formed by the accelerating fields [16].

Consider an array of electric field stages separated by a distance $L$, as shown in Fig. 1. Each stage consists of two parallel cylindrical metal rods with radius $r$, centered at a distance $2 r+d$ apart. One of the rods is connected to a positive and the other to a negative switchable high voltage power supply. Alternating stages are connected to each other. When a molecule in a quantum state with a positive Stark effect (a so-called low-field seeker) moves through the array of electric field stages as indicated in Fig. 1, it will gain Stark energy. This gain in potential energy is compensated by a loss in kinetic energy. If the electric

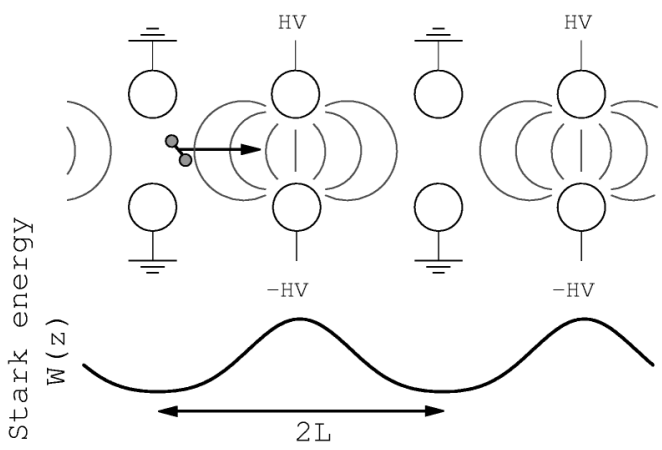

FIG. 1. Scheme of the Stark decelerator, together with the Stark energy of a molecule as a function of position $z$ along the molecular beam axis. 
field is abruptly switched off, the molecule will keep its instantaneous velocity. If we simultaneously switch on the electric field of the next stage the process will repeat itself.

In Fig. 1 the potential energy of the molecule, $W(z)$, is depicted as a function of its position $z$ along the beam axis. The energy a molecule loses per stage depends on its position at the time that the fields are being switched. In analogy with concepts used in charged particle accelerators, we express this position in terms of a "phase angle" $\phi$ which has a periodicity of $2 L$. Molecules which are in maximum electric field just prior to the time at which the fields are being switched are assigned a phase angle of $\phi=90^{\circ}$.

We will first discuss the situation where the electric fields are switched at equal time intervals $\Delta T$. Let us consider a molecule at a phase $\phi=0^{\circ}$ and with a velocity that matches the frequency of the electric fields, i.e., a molecule that travels exactly the distance $L$ in a time interval $\Delta T$. We will refer to this molecule as the "synchronous" molecule (vide infra). Its phase and velocity are indicated as the equilibrium phase $\phi_{0}$ and the equilibrium velocity $v_{0}$, respectively. It is readily seen that (i) the phase and velocity of the synchronous molecules remain unchanged, and (ii) that molecules with a slightly different phase or velocity will experience an automatic correction towards these equilibrium values. A molecule with a phase slightly larger than $\phi_{0}$ and a velocity equal to $v_{0}$, for instance, will lose more energy per stage than the synchronous molecule. It will thus be slowed down relative to the synchronous molecule and consequently its phase will get smaller, until it lags behind. At this point, the situation is reversed and it will lose less energy than the synchronous molecule, etc. This argument shows that molecules with a slightly different phase from $\phi_{0}$ and/or a slightly different velocity from $v_{0}$ will oscillate with both phase and velocity around the equilibrium values; the molecules are trapped in a potential well traveling at the velocity of the synchronous molecule.

In order to decelerate the molecules one has to lower the velocity of the potential well, by gradually increasing the time intervals $\Delta T$ after which the electric fields are being switched. The synchronous molecule will still travel a distance $L$ in the interval $\Delta T$, but $\phi_{0}$ will now be different from zero. By definition, the synchronous molecule is always at the same position when the fields are being switched ( $\phi_{0}$ remains constant); it will achieve this by losing exactly the required kinetic energy per stage. Again, the phase and velocity of a nonsynchronous molecule will oscillate around those of the decelerated synchronous molecule.

The kinetic energy lost by the synchronous molecule per stage, $\Delta K\left(\phi_{0}\right)$, is given by $W\left(\phi_{0}\right)-W\left(\phi_{0}+\pi\right)$. It is convenient to express $W(\phi)$ as a Fourier series. In the expression for $\Delta K\left(\phi_{0}\right)$ all the even terms cancel, yielding $\Delta K\left(\phi_{0}\right)=2 a_{1} \sin \left(\phi_{0}\right)+2 a_{3} \sin \left(3 \phi_{0}\right)+\cdots$. When adjacent electric field stages are not too far apart, i.e., $L \sim$ $2 r+d, \Delta K\left(\phi_{0}\right)$ is predominantly determined by the first term. As mentioned above, the phase is defined only at the moment at which the fields are being switched. To be able to mathematically describe the motion of the molecules through the Stark decelerator, a description in terms of continuous variables is needed. For a description of the motion of a nonsynchronous molecule relative to the motion of the synchronous molecule we introduce the instantaneous difference in phase, $\Delta \phi=\phi-\phi_{0}$, and velocity, $\Delta v=v-v_{0}$. One can regard the lost kinetic energy per stage of the synchronous molecule to originate from a continuously acting, average force $\bar{F}\left(\phi_{0}\right)=-\Delta K\left(\phi_{0}\right) / L$. When $\Delta v \ll v_{0}$, the average force on a nonsynchronous molecule can be written as $\bar{F}\left(\phi_{0}+\Delta \phi\right) \simeq-\Delta K\left(\phi_{0}+\right.$ $\Delta \phi) / L$ [17]. The equation describing the motion of the nonsynchronous molecule relative to the motion of the synchronous molecule is thus given by

$$
\frac{m L}{\pi} \frac{d^{2} \Delta \phi}{d t^{2}}+\frac{2 a_{1}}{L}\left[\sin \left(\phi_{0}+\Delta \phi\right)-\sin \left(\phi_{0}\right)\right]=0
$$

with $m$ the mass of the molecule. This is analogous to the equation for a pendulum driven by a constant torque. For small values of $\Delta \phi$, this results in a harmonic oscillation of $\phi$ around $\phi_{0}$.

In Fig. 2 a numerical integration of Eq. (1) is shown for various equilibrium phases $\phi_{0}$, with parameters as used in the experiment on metastable $\mathrm{CO}$, to be described later. Given a particular equilibrium phase, all molecules positioned in the phase-space diagram inside the corresponding curve will undergo stable phase oscillation. All other molecules will diverge in velocity and phase. The phase stability diagrams showing the longitudinal acceptance of the Stark decelerator are very similar to those used to describe charged particle accelerators (see, for instance, Fig. 13.8 of Ref. [16]). There is an important difference, however. In a charged particle accelerator energy is added to the particles at a certain position, while the amount of energy that is added depends on the time they arrive at this position. In the Stark decelerator energy is added to the molecules at a certain time, while the amount of

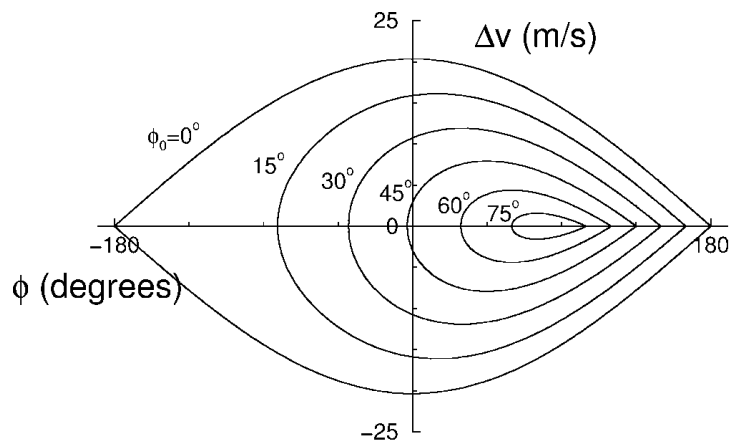

FIG. 2. Phase stability diagrams for various values of $\phi_{0}$, obtained via numerical integration of Eq. (1) with parameters as used in the experiment. 
energy that is added depends on their position at that time. Therefore, while in charged particle accelerators energy and time are conjugate variables, in the Stark decelerator this role is played by velocity (energy divided by velocity) and position (time multiplied by velocity). As a consequence, while the energy spread in the laboratory frame remains constant in a charged particle accelerator, the $v e$ locity spread in the laboratory frame remains constant in the Stark decelerator. The latter is crucial for the promise that the Stark decelerator holds to transfer the high phase-space densities of molecules in the moving frame of the molecular beam to the laboratory frame.

In order to demonstrate the performance of the Stark decelerator, experiments have been carried out on $\mathrm{CO}$ molecules in the $a^{3} \Pi$ state. The main reason to use metastable $\mathrm{CO}$ molecules for these experiments is that (i) they can be prepared at a well-defined position and time, and (ii) their velocity distribution can be readily recorded.

A pulsed beam of $\mathrm{CO}$ is produced by expanding a 5\% $\mathrm{CO}$ in $\mathrm{Xe}$ mixture, using a modified solenoid valve, into vacuum. Cooling the valve housing to $180 \mathrm{~K}$ (just above the boiling point of $\mathrm{Xe}$ ) brings the mean velocity of the $\mathrm{CO}$ molecules in the beam down to $280 \mathrm{~m} / \mathrm{s}$, corresponding to an initial kinetic energy of $E_{\text {kin }}=92 \mathrm{~cm}^{-1}$. The velocity spread is approximately $12 \%$, corresponding to a temperature of $0.48 \mathrm{~cm}^{-1}$ or $\sim 0.7 \mathrm{~K}$ in the moving frame. The $\mathrm{CO}$ molecules pass through a $1.0 \mathrm{~mm}$ diameter skimmer into a second, differentially pumped, vacuum chamber. Preparation of a pulsed beam of metastable CO molecules in single quantum levels is performed by direct laser excitation of the ground state molecules on the spinforbidden $a^{3} \Pi\left(v^{\prime}=0\right) \leftarrow X^{1} \Sigma^{+}\left(v^{\prime \prime}=0\right)$ transition, using pulsed $206 \mathrm{~nm}(6.0 \mathrm{eV})$ radiation. In our experimental setup, the molecules need to be prepared in states that experience a positive Stark effect which are the upper components of the $\Lambda$ doublets in the $a^{3} \Pi$ state [18]. In the experiments reported here, laser preparation of the $J^{\prime}=1 a^{3} \Pi_{1}$ level via the $Q_{2}(1)$ transition is used.

Laser preparation is performed in a $0.3 \mathrm{~mm}$ diameter spot, $7 \mathrm{~mm}$ in front of the $35 \mathrm{~cm}$ long Stark decelerator consisting of an array of 63 equidistant $(L=$ $5.5 \mathrm{~mm}$ ) electric field stages. The two opposing rods $(r=1.5 \mathrm{~mm}, d=2.0 \mathrm{~mm})$ are simultaneously switched by two independent high voltage switches to maximum voltages of $+10 \mathrm{kV}$ and $-10 \mathrm{kV}$. Under these conditions the trap depth is $0.8 \mathrm{~cm}^{-1}$ or $1.15 \mathrm{~K}$. As there is an electric field minimum on the molecular beam axis, there is a net force driving the molecules in the low-field seeking states towards this axis. The pairs of rods of adjacent electric field stages are alternately positioned horizontally and vertically to get focusing of the low-field seeking states in either direction. The actual number of electric field stages that is used can be varied by adjusting the computer controlled sequence by which the switches are triggered. The time-of-flight (TOF) distributions over the $54 \mathrm{~cm}$ distance from laser preparation to detection is recorded by measuring the number of electrons emitted from a flat gold surface when the metastable $\mathrm{CO}$ molecules impinge on it.

In the left part of Fig. 3 the measured TOF distributions are plotted for an increasing number of electric field stages used, as indicated in the figure. The experimental curves, which are given an offset for clarity, are normalized to have the same integrated intensity; the actual intensity increases by more than a factor of 5 with an increasing number of stages used due to transversal focusing effects. The electric fields are switched at equal time intervals of $\Delta T=20 \mu \mathrm{s}$, such that the traveling potential is moving at $275 \mathrm{~m} / \mathrm{s}$, close to the mean velocity of the molecular beam. By varying the number of electrodes used, the time that the trapping potential is on is varied. Therefore, the observed TOF distributions are snapshots of the motion of the metastable $\mathrm{CO}$ molecules in the traveling potential well. The width of the TOF distributions is a convolution of the spatial and velocity distribution of the trapped molecules. However, as the velocity spread of these molecules can be as large as $12 \%$ while the spatial extent of the bunch of molecules is always below $2 \%$ of the flight path, the TOF distribution mainly reflects the velocity distribution. The oscillation of the width of the TOF distributions with increasing number of stages, therefore, directly reflects the oscillation in the velocity spread of the trapped molecules. The dashed curve above the TOF distribution recorded with 47 stages results from a Monte Carlo simulation using Eq. (1). On the right-hand side of Fig. 3

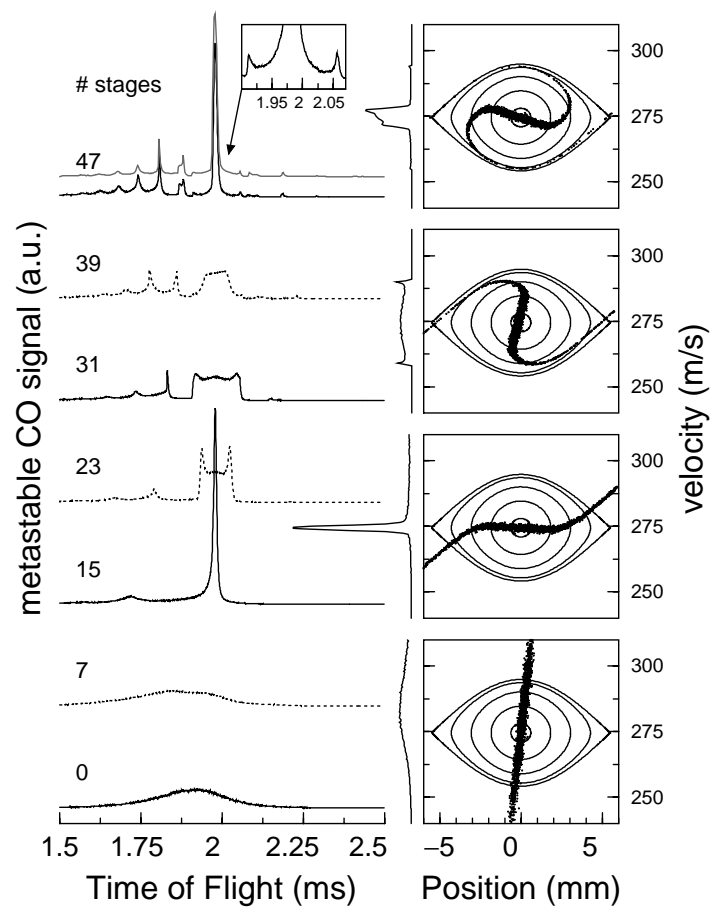

FIG. 3. Observed TOF distributions using an increasing number of electrodes for $\phi_{0}=0^{\circ}$. On the right, the results from Monte Carlo calculations are plotted in phase-space diagrams, when $0,15,31$, and 47 stages are used. 

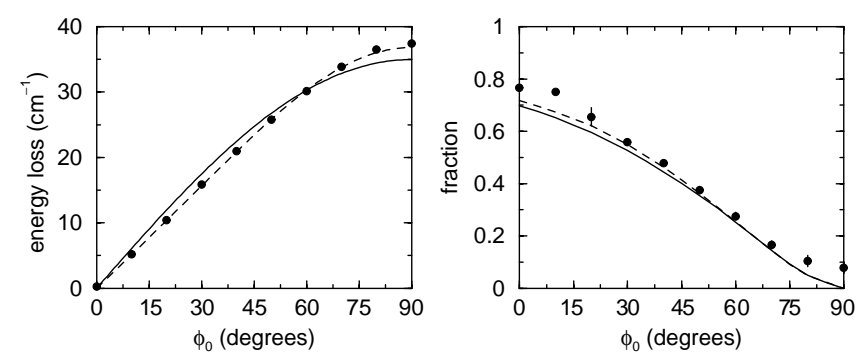

FIG. 4. Observed kinetic energy loss over 47 stages (left) and the observed fraction of the molecular beam that is decelerated (right) as a function of $\phi_{0}$, compared to results from the analytical model.

the results from the simulation for $0,15,31$, and 47 electric field stages, are plotted in phase-space diagrams, together with several (including the outermost) orbits of the phase stability diagram for $\phi_{0}=0^{\circ}$. The position of a molecule in phase space is represented by a dot in these diagrams. The projections of the distributions of molecules in phase space onto the velocity axis, as shown on the left axes, give the true velocity distributions of the trapped molecules. Since the metastable molecules are prepared in a $0.3 \mathrm{~mm}$ diameter laser focus, only a small region in phase space is occupied at the entrance of the Stark decelerator. This enables the direct observation of the oscillatory motion of the molecules in velocity and position. The molecules are seen to describe closed orbits in the position-velocity plane. Because of the anharmonicity of the traveling potential well the outer orbits have longer periods than the inner ones, resulting in spiral structures. The tails of these spirals are seen as sharp edges in the upper TOF distribution and are shown in more detail in the inset. The narrowest velocity distribution results when the distribution of the molecules in the phase-space diagrams is "horizontal." The ultimate width of the velocity distribution is determined by the initial spatial extent of the bunch of molecules at the entrance of the Stark decelerator. When 15 stages are used, the relative velocity spread is $0.7 \%$, corresponding to a temperature in the moving frame of $T=4 \mathrm{mK}$.

The molecules trapped in the potential well can be decelerated by gradually increasing $\Delta T$, at the cost of a decrease of the depth of the potential. In Fig. 4 the experimentally observed fraction of the beam that is confined in the traveling potential well (right) is shown together with the kinetic energy loss of the metastable $\mathrm{CO}$ molecules over 47 stages (left) as a function of $\phi_{0}$. The solid curves show the result using the analytical model with $2 a_{1}=0.76 \mathrm{~cm}^{-1}$. Including the next term in the Fourier expansion with $2 a_{3}=-0.040 \mathrm{~cm}^{-1}$ yields the dashed curves.
The results presented in this Letter clearly demonstrate the potential of the Stark decelerator for transferring the high initial phase-space densities from the moving molecular frame to the laboratory frame. The record-low molecular beam temperatures with their variable velocities achieved in this study hold great promise for a variety of molecular beam experiments.

This work is part of the research program of the "Stichting voor Fundamenteel Onderzoek der Materie (FOM)," which is financially supported by the "Nederlandse Organisatie voor Wetenschappelijk Onderzoek (NWO)." We acknowledge the expert technical assistance of Ch. Timmer.

[1] S. Chu, Rev. Mod. Phys. 70, 685 (1998); C. N. CohenTannoudji, ibid. 70, 707 (1998); W. D. Phillips, ibid. 70, 721 (1998).

[2] J. M. Doyle and B. Friedrich, Nature (London) 401, 749 (1999).

[3] J. D. Weinstein, R. deCarvalho, T. Guillet, B. Friedrich, and J. M. Doyle, Nature (London) 395, 148 (1998).

[4] B. Friedrich, R. deCarvalho, J. Kim, D. Patterson, J. D. Weinstein, and J. M. Doyle, J. Chem. Soc. Faraday Trans. 94, 1783 (1998).

[5] J. T. Bahns, P. L. Gould, and W. C. Stwalley, Adv. At. Mol. Opt. Phys. 42, 171 (2000).

[6] H. L. Bethlem, G. Berden, and G. Meijer, Phys. Rev. Lett. 83, 1558 (1999).

[7] J. A. Maddi, T. P. Dinneen, and H. Gould, Phys. Rev. A 60, 3882 (1999).

[8] M. Gupta and D. Herschbach, J. Phys. Chem. A 103, 10670 (1999).

[9] B. Friedrich, Phys. Rev. A 61, 025403 (2000).

[10] J. G. King, in Proceedings of the 13th Annual Symposium on Frequency Control, Asbury Park, 1959 (U.S. Army Signal Research and Development Laboratory, Fort Monmouth, 1959), p. 603.

[11] R. Wolfgang, Sci. Am. 219, No. 4, 44 (1968).

[12] E. Maréchal, S. Guibal, J.-L. Bossennec, R. Barbé, J.-C. Keller, and O. Gorceix, Phys. Rev. A 59, 4636 (1999).

[13] L. Niel and H. Rauch, Z. Phys. B 74, 133 (1989).

[14] V. I. Veksler, J. Phys. (Moscow) 9, 153 (1945)

[15] E. M. McMillan, Phys. Rev. 68, 143 (1945).

[16] S. Humphries, Jr., in Principles of Charged Particle Accelerators (Wiley, New York, 1986).

[17] More elaborate Monte Carlo calculations, using the true force acting on the molecules upon passage through the Stark decelerator, indicate that breakdown of this approximation when $v_{0}$ becomes small leads only to minor deviations from the here presented model.

[18] R. T. Jongma, G. von Helden, G. Berden, and G. Meijer, Chem. Phys. Lett. 270, 304 (1997). 